\title{
Local global method for the prediction of surface residual stresses in 3D turning
}

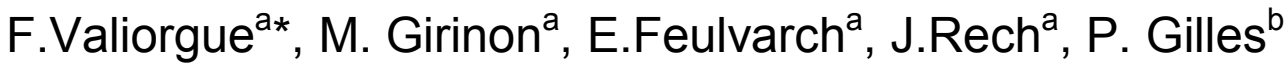 \\ a Univ. de Lyon, ENISE, LTDS, UMR5513 CNRS, 58 rue Jean Parot 42023 Saint Etienne, France \\ ${ }^{b}$.AREVA NP Tour AREVA, 92084 Paris La Défense, France
}

Keywords: Turning; 3D local global simulation; residual stresses.

\begin{abstract}
Numerical simulation of turning is still one of the best solutions to understand and improve such a process. Since many years, researchers have tried to use several numerical approaches to go round the difficulties and to set up reliable models (Lagrangian, ALE,...). Currently no perfect complete solution is available and it is time to introduce dedicated models prone to simulate partially the phenomena in order to reach specific conditions linked with real industrial problematics. This paper will present a 3D local global method set up to predict surface residual stresses in finish turning. This approach uses two kinds of simulations. A first one that allows reaching thermo mechanical steady state around the cutting edge and the chip area. A second one which sequences the application of the extracted thermo mechanical fields onto the real workpiece surface. The obtained results concerning the residual stresses fields will then be compared with the ones recorded experimentally.
\end{abstract}

\section{Introduction}

Since several years, researchers have built numerical models in 2D or 3D to reproduce material removal processes in an attempt to understand cutting mechanisms and induced consequences on the workpiece. Historically, the simulations began with 2D Lagrangian models followed by ALE models [1]. More recently, extruded ALE 2D models and true 3D model have been proposed [2]. These modeling methods are currently dependent on damage laws and remeshing technics and the simulated time is too long to reach thermal and mechanical equilibrium [3]. In this way, hybrid method is a very effective approach to compute induced consequences on the parts but it is still massively dependent on experimental tests [4] [5]. In all this approaches, major drawbacks remain and the transfer into industry is still a challenge. To get usable method, the aim of this work is to propose an approach taking the best of each numerical simulation.

\section{Local global method}

The prediction of residual stresses generated by turning needs two different scales of simulation to be relevant. First, the phenomena occurring around the cutting edge need to be well reproduced. To do so, a local simulation at micro scale is performed and it considers the chip, the tool and the workpiece involved in a steady state single cutting operation. This step allows reproducing thermomechanical consequences applied on the surface workpiece by the cutting operation. Secondly, the thermomechanical consequences are propagated on the workpiece surface so as to reproduce the effects of several passes and to compute the final mechanical equilibrium. This global simulation is performed at meso scale.

Local modeling. The local modeling of turning is based on an Eulerian simulation implemented in SYSWELD ${ }^{\circledR}$. This way of modeling enables to reach a steady state for the material flow (mechanical) and temperatures (like after an infinite time of simulation) and the thermomechanical consequences applied to the workpiece surface are also stabilized. In exchange, this approach needs to mesh the chip "while flowing". For this first approach of the method, only one set of cutting parameters was investigated to machine AISI 304L. The cutting conditions are: 
- Cutting speed: $100 \mathrm{~m} / \mathrm{min}$

- Feed: $0.3 \mathrm{~mm} / \mathrm{rev}$

- Depth of cut: $0.3 \mathrm{~mm}$

The cutting tool is a TiN coated tungsten carbide.

Mesh. In order to build the mesh, Quick Stop Tests are performed allowing catching the chip geometry during the cutting operation. After these tests, the main geometrical characteristics are used to mesh the chip and the material around it (figure 1).

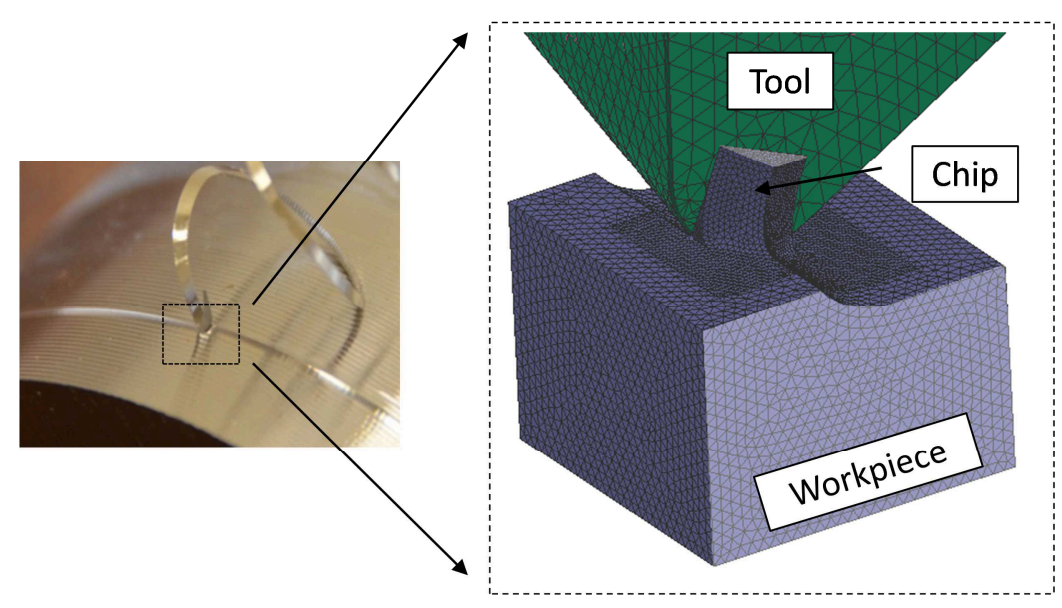

Figure 1: Chip after Quick Stop Test and associated mesh

This model uses tetrahedral elements with the P1+/P1 formulation which enables solving the incompressible viscous flow [6] [7].[8]

Steady state simulation. In this model, viscous stresses are predominant compared to the inertia effects and a Stokes assumption is used for the modeling of the material flow in the chip. The resolution of the physical problem is found by solving the heat equation and the momentum balance. More details of the model are proposed here: [9].

The Eulerian formalism allows computing the material flow around the cutting edge. The figure 3 shows the isovalues of the temperature and the velocity obtained for the industrial conditions.

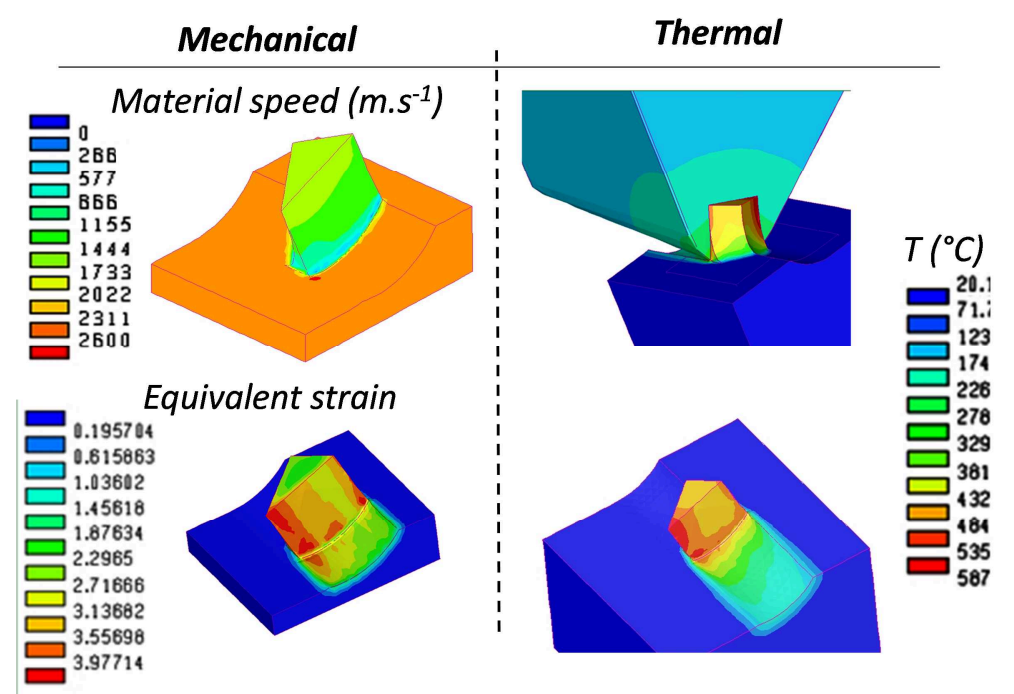

Figure 3: Steady state mechanical and thermal results.

Concerning the thermal properties of the model, it has been set up experimentally and all the details are given in [10]. 


\section{Global modeling}

The thermal and mechanical results generated by the material removal and responsible of the surface alteration are extracted at a plane corresponding to the final surface of the workpiece. These fields are fitted with numerical curves so as to be implemented in the global model (figure 4).

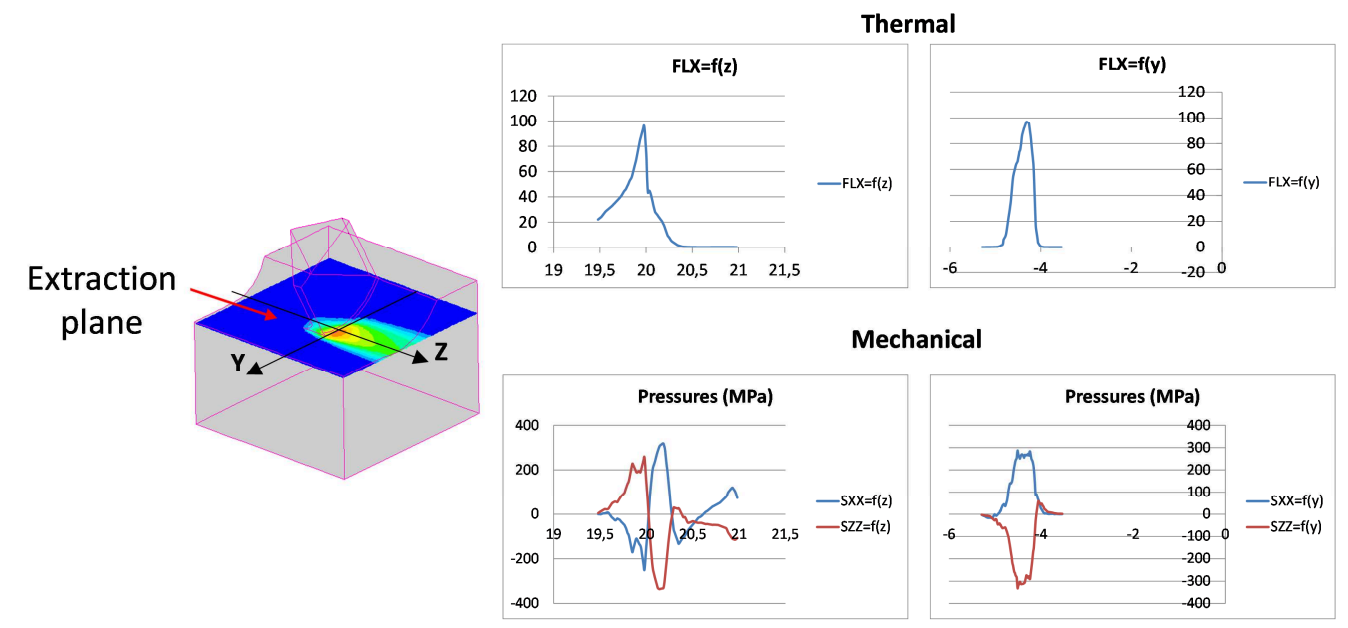

Figure 4: Analytical extraction of thermo mechanical loadings applied to the workpiece surface All the details concerning the propagation model are given in [5]. The figure 5 illustrates the fitted analytical stresses applied to the model.

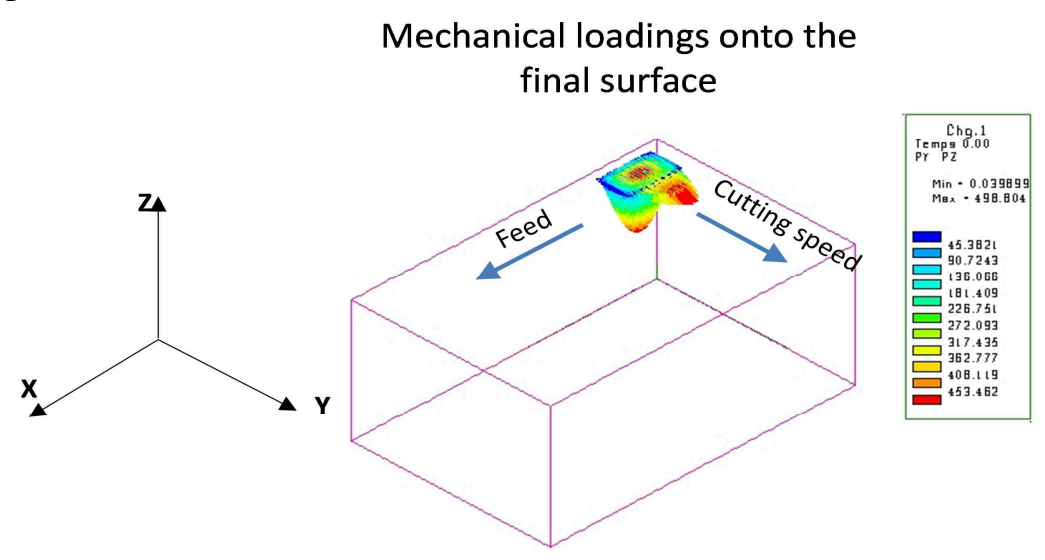

Figure 5: Analytical loadings moved onto the workpiece surface.

\section{Results}

As the main characteristic of the global method is to reach the steady state in the residual stress generation process, results are extracted in the steady zone (figure 6). 

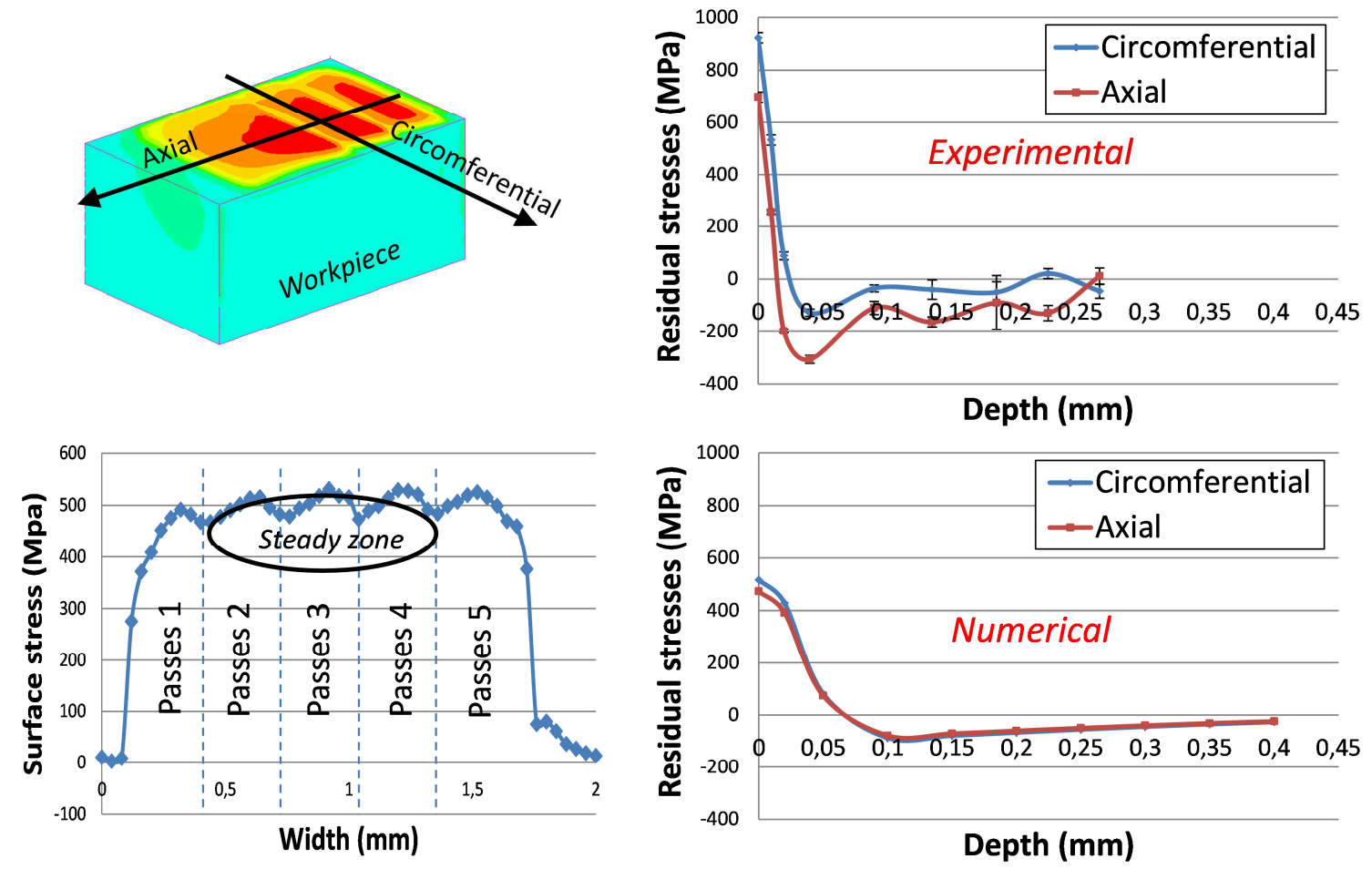

Figure 6: Results.

It appears that the numerical results have the good shape for the residual stress gradient but the values are quite different between experimental and numerical curves. The top surface residual stress, the maximum compressive value and the affected depth are not matching very well for both directions. This may be due to lack of precision in the extracted loadings coming from the local model. In fact, mechanical field pressure should be thinner in order to concentrate the affected zone in the first tenth of millimeter and intensify the compression pick. These are ongoing developments and results should be presented later.

\section{Conclusions}

The paper presents an original method to predict residual stresses fields and more generally surface integrity. It is based on a local-global approach which tries to retain only the relevant characteristics of two methods of simulation. The local model, based on Eulerian formulation, reproduces finely the material flow around the cutting edge in steady state and the global model sequences the thermomechanical affectations onto the workpiece surface. The global model also computes the final mechanical equilibrium responsible of the residual stresses. Nevertheless, the results in terms of residual stresses have to be improved. In fact the size and the intensity of the computed loadings are not close enough the reality and do not provide relevant input data for the global model. As the global model has shown its possibilities in other studies, the developments and improvements on the Eulerian model will be the priority of the future works. 


\section{References}

[1] L.-J Xie, J. Schmidt, C. Schmidt, F. Biesinger, 2D FEM estimate of tool wear in turning operation, Wear. 258 (2005) 1479-1490 Issue 10.

[2] P.J. Arrazola, A. Kortabarria, A. Madariaga, J.A. Esnaola, E. Fernandez, C. Cappellini,D. Ulutan, T. Özel, On the machining induced residual stresses in IN718 nickel-based alloy: Experiments and predictions with finite element simulation, Simulation Modelling Practice and Theory. 40 (2014) 87-103,

[3] E. Ceretti, L. Filice, D. Umbrello, F. Micari, ALE Simulation of Orthogonal Cutting: a New Approach to Model Heat Transfer Phenomena at the Tool-Chip Interface, CIRP Annals Manufacturing Technology. 56 (2007) 69-72 Issue 1

[4] A. Mondelin, F. Valiorgue, J. Rech, M. Coret, E. Feulvarch, Hybrid model for the prediction of residual stresses induced by 15-5PH steel turning, International Journal of Mechanical Sciences. 58 (2012) 69-85 issue 1

[5] F. Valiorgue, J.Rech, H. Hamdi, P. Gilles, J.M. Bergheau, 3D modeling of residual stresses induced in finish turning of an AISI304L stainless steel, International Journal of Machine Tools and Manufacture. 53 (2012) 77-90 issue 1

[6] D.N. Arnold, F. Brezzi, M. Fortin, A stable element for the Stokes equations, Calcolo. 21 (1984) 337-344.

[7] T. Heuzé, J.B. Leblond, J.M. Bergheau, E. Feulvarch, A finite element for laminar flow of incompressible fluids with inertia effects and thermo mechanical coupling, European Journal of Computational Mechanics. 19 (2010) 293-304.

[8] E. Feulvarch, N. Moulin, P. Saillard, T. Lornage, J.-M. Bergheau, 3D simulation of glass forming process Original Research Article, Journal of Materials Processing Technology. 164-165 (2005) 1197-1203

[9] E. Feulvarch, J.-C. Roux, J.-M. Bergheau, A simple and robust moving mesh technique for the finite element simulation of Friction Stir Welding, Original Research Article, Journal of Computational and Applied Mathematics. 246 (2013) 269-277

[10] A. Mondelin, F. Valiorgue, E. Feulvarch, J. Rech, M. Coret, Calibration of the insert/tool holder thermal contact resistance in stationary 3D turning. Applied Thermal Engineering. 55 (2013) $17-25$ 